\title{
Chemotherapie bei prämenopausalen Krebspatientinnen: Erhalt der Ovarialfunktion durch Goserelin
}

Mammakarzinome werden zunehmend bei jüngeren Frauen diagnostiziert: Aktuellen Daten zufolge ist rund ein Drittel aller Brustkrebspatientinnen - das sind allein in Deutschland über 15000 Frauen pro Jahr - prämenopausal, berichtete im Mai 2003 Prof. Dr. Bernd Gerber von der I. Frauenklinik der LMU München auf einem Symposium von AstraZeneca. Dank der heute praktizierten aggressiveren Therapie haben sie einerseits eine hohe Heilungschance, müssen aber andererseits im weiteren Verlauf mit den Langzeitfolgen der antitumoralen Therapie leben. Dies gilt in gleicher Weise für junge Frauen mit anderen Tumorerkrankungen, z. B. den gerade bei Jugendlichen häufigen hämatologischen Neoplasien: Hier führt die intensive
Zytostatikatherapie in rund $70 \%$ der Fälle zu einem Ausfall der Ovarfunktion und einer dauerhaften Amenorrhoe.

\section{Negative Langzeitfolgen der Chemotherapie bei jungen Frauen}

Diese Frauen sind zwar geheilt, können aber nicht schwanger werden. Darüber hinaus werden sie lebenslang mit den Folgen des vorzeitigen Ausfalls der Östrogenproduktion zu kämpfen haben: Sie leiden nicht nur an den Beschwerden der vorzeitigen Menopause wie Hitzewallungen oder Schlafstörungen, sondern weisen zudem ein deutlich erhöhtes kardiovas-

Tab. 1. Aktuelle Studien zum präventiven und adjuvanten Effekt des Hormonentzugs bei prämenopausalen Patientinnen

\begin{tabular}{|c|c|c|c|c|c|c|}
\hline Autor (Jahr) & $\begin{array}{l}\text { Patientinnen- } \\
\text { charakteristik }\end{array}$ & $\begin{array}{l}\text { Follow-up } \\
\text { (Monate) }^{\mathrm{a}}\end{array}$ & $\begin{array}{l}\text { Randomisiert } \\
\mathrm{zu}:\end{array}$ & $\mathrm{n}$ & Ereignisse & $\begin{array}{l}\text { Rel. Risiko } \\
(95 \%-C I)\end{array}$ \\
\hline Love (2002) & $\begin{array}{l}\text { Asiatinnen, } \\
\text { ER? }\end{array}$ & 42 & $\begin{array}{l}\text { Beobachtung } \\
B S O+\text { Tam }\end{array}$ & $\begin{array}{l}353 \\
356\end{array}$ & $\begin{array}{l}127(36 \%) \\
84(24 \%)\end{array}$ & $\begin{array}{l}1.0 \\
\text { DFS: } 0,58(0,4-0,8) \\
\text { OS: } 0,67(0,4-1,0)\end{array}$ \\
\hline Kauff (2002) & BRCA1/2+ & 24 & $\begin{array}{l}\text { Beobachtung } \\
B S O\end{array}$ & $\begin{array}{l}72 \\
98\end{array}$ & $\begin{array}{l}8(11 \%) \\
3(3 \%)\end{array}$ & $\begin{array}{l}1.0 \\
0,32(0,08-1,20)\end{array}$ \\
\hline $\begin{array}{l}\text { Rebbeck } \\
(2002)\end{array}$ & BRCA1/2+ & 108 & $\begin{array}{l}\text { Beobachtung } \\
B S O\end{array}$ & $\begin{array}{l}142 \\
99\end{array}$ & $\begin{array}{l}60(42 \%) \\
21(21 \%)\end{array}$ & $\begin{array}{l}1,0 \\
0,47(0,29-0,77)\end{array}$ \\
\hline
\end{tabular}

${ }^{\mathrm{a}}$ Median.

$\mathrm{CI}=$ Confidence interval; ER = Östrogenrezeptor; BSO = Bilaterale Salpingo-Oopherektomie; Tam = Tamoxifen DFS $=$ Disease-Free Survival; OS = Overall Survival.

Tab. 2. ZEBRA-Studie: Einfluss der CMF-induzierten Amenorrhoe auf krankheitsfreies Überleben und Knochendichte

\begin{tabular}{|c|c|c|c|c|c|c|}
\hline \multirow[t]{2}{*}{ Faktor } & \multicolumn{3}{|c|}{ DFS } & \multicolumn{3}{|l|}{ OS } \\
\hline & RR & $95 \%-\mathrm{CI}$ & p-Wert & $\mathrm{RR}$ & $95 \%-\mathrm{CI}$ & p-Wert \\
\hline \multicolumn{7}{|l|}{ Tumorgröße } \\
\hline $\mathrm{T} 11$ & 1 & & & 1 & & \\
\hline $\mathrm{T} 2, \mathrm{~T} 3$ & 1,9 & $1,5-5,1$ & 0,03 & 3,3 & $1,3-8,5$ & 0,01 \\
\hline \multicolumn{7}{|l|}{ Nodaler Status } \\
\hline $1-3$ & 1 & & & 1 & & \\
\hline$\geq 4$ & 2,8 & $1,1-3,5$ & 0,001 & 2,4 & $1,0-5,8$ & 0,04 \\
\hline \multicolumn{7}{|l|}{ Menstruationsstatus } \\
\hline Ovariale Dysfunktion & 1 & & & 1 & & \\
\hline Regelmäßige Menstruation & 2,1 & $1,2-4,2$ & 0,01 & 2,2 & $0,9-5,2$ & 0,09 \\
\hline \multicolumn{7}{|l|}{ ER-/PR-Status } \\
\hline Positiv & & & & 1 & & \\
\hline Negativ & & & & 4,0 & $1,6-9,8$ & $<0,005$ \\
\hline
\end{tabular}

DFS = Disease-Free Survival; OS = Overall Survival; RR = Relatives Risiko CI = Confidence Interval; $\mathrm{ER}=$ Östrogenrezeptor; $\mathrm{PR}=$ Progesteronrezeptor kuläres Risiko auf. Nicht zu unterschätzen ist auch die Frakturgefährdung aufgrund der früh einsetzenden Osteoporose. Bei Brustkrebspatientinnen wird von einer späteren Hormonersatztherapie zur Prävention dieser Beschwerden und Risiken ohnehin in der Regel abgeraten. Aber auch bei Patientinnen mit nicht hormonabhängigen Tumoren erscheint eine langfristige Hormonsubstitution mittlerweile aufgrund neuer Daten, z.B. aus der HERS-Studie und der Women's Health Initiative (WHI), zu risikoreich und kann heute nicht mehr empfohlen werden, warnte Gerber.

Grundsätzlich kann bei prämenopausalen $\mathrm{Pa}$ tientinnen mit rezeptorpositivem Brustkrebs nicht auf einen Hormonentzug verzichtet werden. Darauf verweisen mehrere Studien, in denen eine in präventiver oder therapeutischer Intention erfolgte Ovarektomie das Risiko für die Entwicklung eines Mammakarzinoms bzw. das Rezidivrisiko dramatisch reduzierte (Tab. 1). Auch der Nutzen einer adjuvanten Chemotherapie bei prämenopausalen Brustkrebspatientinnen wird in erster Linie auf die Suppression der Ovarialfunktion und weniger auf den zytotoxischen Effekt zurückgeführt. Allerdings variieren die Amenorrhoeraten je nach eingesetztem Regime stark, liegen z.B. im Falle des CMF(Cyclophosphamid, Methotrexat, 5-Fluorouracil)-Regimes bei weit über $60 \%$, bei anthrazyklinhaltigen Regimen dagegen nur zwischen 30 und 40\%. Die Prognose der zytostatisch behandelten Patientinnen hängt stark davon ab, ob die Induktion einer Amenorrhoe gelingt. Das demonstriert die ZEBRA(Zoladex Early Breast Cancer Research Association)-Studie, in der das CMF-Regime mit dem GnRH-Agonisten Goserelin $\left(\right.$ Zoladex $\left.^{\circledR}\right)$ bei 1640 prämenopausalen Brustkrebspatientinnen verglichen wurde: Weiterhin menstruierende Frauen des CMFArms wiesen gegenüber den amenorrhoeischen Frauen ein rund dreifach erhöhtes Rezidivrisiko auf. Allerdings zahlten die amenorrhoeischen Patientinnen für die bessere Prognose einen hohen Preis: Bereits innerhalb des ersten Studienjahres war ein massiver Abbau an Knochenmasse festzustellen, der nach Beendigung der Chemotherapie nicht reversibel war (Tab. 2). Eine präventive Bisphosphonat-Gabe ist bei diesen Frauen wenig effektiv: Die Abnahme der Knochendichte fällt bei mit Clodronat behandelten Frauen zwar geringer aus als bei unbehandelten Kontrollen, ist aber dennoch sehr viel ausgeprägter als bei nach Therapieende wieder menstruierenden Frauen, wie Vehmanen et al. kürzlich zeigen konnten.

\section{Endokrine Kombination schlägt Standard-Chemotherapie}

Als effektive und verträglichere Alternative zur Chemotherapie bei rezeptorpositivem Brustkrebs in der Prämenopause betrachtet Gerber die endokrine Therapie mit einem GnRH-Agonisten wie Goserelin, das zusätzlich noch mit dem Antiöstrogen Tamoxifen kombiniert werden kann. In der ZEBRA-Studie erwies sich die Goserelin-Therapie beim hormonsensitiven Brustkrebs hinsichtlich krankheitsfreiem und 
Gesamt-Überleben als ebenso effektiv wie das CMF-Regime. In der österreichischen Studie AC05 der Austrian Breast and Colorectal Cancer Study Group war die Kombination aus Goserelin/Tamoxifen dem CMF-Regime beim krankheitsfreien Überleben sogar signifikant, beim Gesamt-Überleben tendenziell überlegen. Auch mit anthrazyklinhaltigen Regimen zeigten sich keine Wirksamkeitsvorteile im Vergleich zur endokrinen Therapie - nicht verwunderlich angesichts der doch relativ niedrigen Amenorrhoeraten dieser Schemata. Laut Prof. Dr. Raimund Jakesz, Wien, ist bei jungen Patientinnen mit nodalnegativem Brustkrebs die Monotherapie mit einem GnRH-Agonisten eine mögliche Option. Dagegen sollte bei positivem Nodalstatus immer der Kombination mit GnRH-Agonist plus Tamoxifen oder der Sequenz Chemotherapie gefolgt von Tamoxifen der Vorzug gegeben werden.

Das große Plus der Hormontherapie mit Goserelin ist die zeitliche Begrenzung des Hormonentzugs, betonte Gerber. Nach Beendigung der Goserelin-Behandlung setzt die Menstruation bei vielen Frauen wieder ein, ohne dass sich dies ungünstig auf die weitere Prognose auswirkt: Entsprechend war das krankheitsfreie Überleben von dauerhaft amenorrhoeischen und wieder menstruierenden Frauen in der ZEBRA-Studie vergleichbar. Zwar nahm die Knochendichte der mit Goserelin behandelten Frauen unter der Therapie - ähnlich wie im CMF-Arm - ebenfalls ab. Nach Absetzen von Goserelin und wieder einsetzender Menstruation ist dieser Verlust jedoch reversibel. Auch die zuvor aufgetretenen Wechseljahrsbeschwerden gehen zurück.

\section{Goserelin wirkt ovarialprotektiv}

Als wichtigen, aber bislang zu wenig gewürdigten Aspekt der Goserelin-Therapie nannte Gerber den Schutz der ovariellen Funktion durch den GnRH-Agonisten, der insbesondere bei zytostatisch behandelten Frauen zum Tragen kommt. Da Goserelin die Follikelreifung unterdrückt, wird eine Schädigung von DNA und Enzymsystemen in den Follikelzellen durch die Chemotherapie verhindert und die Ovarialfunktion langfristig gesichert (Abb. 1).

Dieser protektive Effekt von Goserelin wurde in einer italienischen Studie an 64 prämenopausalen Patientinnen mit großen Mammakarzinomen (T2-3) belegt. 28 Frauen mit rezeptorpositiven Tumoren wurden zusätzlich zur Chemotherapie mit Goserelin/Tamoxifen behandelt, 36 Frauen mit negativem Rezeptorstatus erhielten Goserelin für 1 Jahr begleitend zur Chemotherapie. Bei allen Frauen sanken die Hormonspiegel im Therapieverlauf auf postmenopausale Werte ab. Gerber bezeichnete die Gesamtund tumorfreie Überlebensrate mit 94 bzw. $84 \%$ nach 55-monatigem Follow-up in diesem Kollektiv angesichts der ausgedehnten Tumoren als hoch. Nach Abschluss der Therapie traten bei $86 \%$ der Frauen wieder Menstruationen auf. Selbst mehrere normal verlaufende Schwangerschaften wurden registriert.

«Zur Prävention einer dauerhaften Amenorrhoe sollte deshalb 1-2 Wochen vor Beginn einer Chemotherapie ein GnRH-Agonist verabreicht werden», empfahl Gerber. Nach Beendingung der Zytostatikatherapie sollte die endokrine Therapie noch über mindestens einen Menstruationszyklus hinweg fortgesetzt werden. Jakesz bewertete einen derartigen Ovarschutz durch Goserelin als «faszinierendes Konzept für junge Frauen, die nach kurativer Chemotherapie schwanger werden wollen».

Laut Gerber sind Schwangerschaften bei jungen Frauen nach Mammakarzinom nicht generell als kontraindiziert anzusehen. Vielmehr weisen einige Studien darauf hin, dass die Langzeitprognose - aus bislang noch ungeklärten Gründen - eher günstig beeinflusst wird. Allerdings sollte eine Schwangerschaft wegen des in den ersten 2-3 Jahren erhöhten Rezidiv- und Metastasierungsrisikos nicht sofort nach Abschluss der Therapie geplant werden.

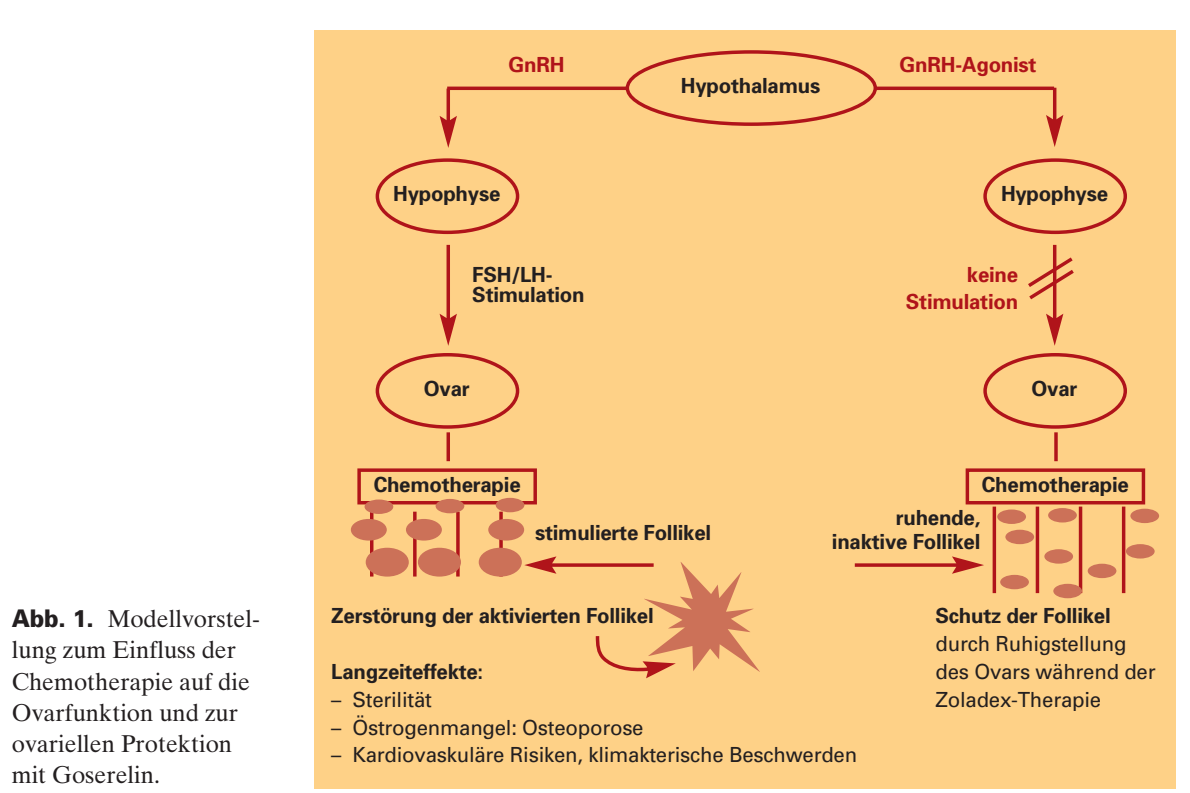

\section{Ovarialschutz auch bei systemischen Neoplasien}

Noch viel zu selten umgesetzt wird dieses Konzept bei sehr jungen Frauen mit hämatologischen Systemerkrankungen, kritisierte Gerber. Sie können dank der heute möglichen Therapie in der Mehrzahl der Fälle geheilt werden. Bei diesen sehr jungen Patientinnen mit zumeist bestehendem Kinderwunsch ist der Erhalt der Ovarialfunktion besonders vordringlich, zumal die Tumoren nicht hormonabhängig sind. Der präventive Einsatz von GnRH-Agonisten wurde mittlerweile in einer prospektiven Studie erfolgreich geprüft: Während die unbehandelten Kontrollpatientinnen nach Abschluss der Chemotherapie in knapp $60 \%$ der Fälle dauerhaft amenorrhoeisch blieben, trat dank des prophylaktischen Einsatzes von GnRH-Agonisten bei 94\% der so Behandelten wieder eine normale Menses auf.

\section{Weiterführende Literatur}

Jonat W, Kaufmann M, Sauerbrei R, et al.: J Clin Oncol 2002; 20:4628-4635.

Vehmanen L, Saarto T, Elomaa I, et al.: Eur J Cancer 2001; 37:2373-2378.

Jakesz R, Hausmaninger H, Kubista E, et al.: J Clin Oncol 2002; 20:4621-4627.

Recchia F, Sica G, de Filippi S, et al.: Anticancer Drugs 2002; $13 \cdot 417-424$

Blumenfeld Z: Mol Cell Endocrinol 2002;187: 93- 105.

\section{Quelle}

Symposium «Pioneering Progress in Breast Cancer Management», Valencia, Spanien, 16.-18. Mai 2003.

Bericht: Dr. Katharina Arnheim, Berlin

\section{Impressum}

Chemotherapie bei prämenopausalen Krebspatientinnen: Erhalt der Ovarialfunktion durch Goserelin

PharmaForum in Onkologie 26 | 4 | 03

(c) 2003 by S. Karger Verlag

Verlag für Medizin und Naturwissenschaften $\mathrm{GmbH}$

Lörracher Straße 16a

D-79115 Freiburg

Tel. +49761452070

E-mail Information@Karger.de

Mit freundlicher Unterstützung der AstraZeneca GmbH.

Der Verlag und die Herausgeber der Zeitschrift übernehmen keine Verantwortung für diese Rubrik.

Die Wiedergabe von Gebrauchsnamen, Handelsnamen, Warenbezeichnungen usw. in dieser Zeitschrift berechtigt auch ohne besondere Kennzeichnung nicht zur Annahme, dass solche Namen im Sinne der Warenzeichen- und Markenschutz-Gesetzgebung als frei zu betrachten wären und daher von jedermann benutzt werden dürfen. Für Angaben von Dosierungsanweisungen und Applikationsformen kann vom Verlag keine Haftung übernommen werden. Derartige Angaben müssen vom jeweiligen Anwender im Einzelfall anhand anderer Literaturstellen auf ihre Richtigkeit überprüft werden. 